\title{
Left atrial reservoir function predicts atrial fibrillation recurrence after catheter ablation: a two-dimensional speckle strain study
}

\author{
Mahek Mirza, Giuseppe Caracciolo, Uzma Khan, Naoyo Mori, Samir K. Saha, Komandoor \\ Srivathsan, Gregory Altemose, Luis Scott, Partho Sengupta, and Arshad Jahangir \\ Division of Cardiovascular Diseases, Mayo Clinic, 13400 East Shea Boulevard, Scottsdale, AZ \\ 85259, USA
}

\begin{abstract}
Background-Predictors of atrial fibrillation (AF) recurrence after catheter ablation (CA) are not fully defined. We hypothesized that 2D left atrial (LA) regional strain maps would help identify abnormal atrial substrate that increases susceptibility to AF recurrence post-CA.

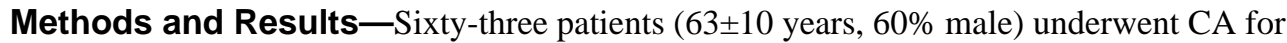
symptomatic paroxysmal (75\%) or persistent (25\%) AF. Baseline LA mechanical function determined using speckle tracking echocardiography was compared between those with $\mathrm{AF}$ recurrence (AFR) and no recurrence post-CA. Bi-dimensional global and regional maps of LA wall velocity, strain, and strain rate (SR) were obtained during end ejection and early diastole. After $18 \pm 12$ months of follow-up, 34 patients were free of AFR post-CA. There were no differences in clinical characteristics, LA and LV volumes, and Doppler estimates of LV diastolic function and filling pressures at baseline between patients with recurrent $\mathrm{AF}$ and those that maintained sinus rhythm. However, the LA emptying fraction $(55 \pm 17 \%$ vs. $64 \pm 14 \%, p=0.04)$, global and regional systolic and diastolic strains, SR, and velocities were reduced in patients with recurrent AF. There was marked attenuation of peak LA lateral wall longitudinal strain (LS; $11 \pm 7 \%$ vs. $20 \pm 14 \%, p=0.007)$ and SR $\left(0.9 \pm 0.4\right.$ vs. $\left.1.3 \pm 0.6 \mathrm{~s}^{-1}, p=0.01\right)$. Multivariate analysis revealed lateral wall $\mathrm{LS}$ (odds ratio $=1.15,95 \% \mathrm{CI}=1.02-1.28, p=0.01$ ) as an independent predictor of AFR.
\end{abstract}

Conclusions-Regional LA lateral wall LS is a pre-procedural determinant of AFR in patients undergoing CA, independent of LA enlargement. Characterization of atrial myocardial tissue properties by speckle tracking echo may aid the appropriate selection of adjunctive strategies and prognostication of patients undergoing CA.

\section{Keywords}

Atrial fibrillation; Catheter ablation; Strain and strain rate; Atrial size

\begin{abstract}
Atrial fibrillation (AF) is a heterogeneous disorder with variable etiologies and natural history depending on the age of onset and presence of underlying heart disease [1-3]. Structural and functional changes in the atrial myocardium with advancing age and presence of comorbidities lead to regional impairment of impulse generation and conduction that contributes to progressive atrial remodeling constituting an abnormal substrate that renders
\end{abstract}

\footnotetext{
(C) Springer Science+Business Media, LLC 2011

Correspondence to: Arshad Jahangir.

jahangir.arshad@mayo.edu .

Mahek Mirza and Giuseppe Caracciolo both contributed equally to the work.

Disclosures None.
} 
AF refractory to therapeutic interventions $[1,4]$. Catheter ablation (CA) by elimination of arrhythmogenic foci and modification of atrial structural substrate that promotes AF is effective in restoring sinus rhythm and appears to be superior to anti-arrhythmic therapy in preventing recurrence of AF in a majority of patients [5-9]. Despite recent advances in techniques for $\mathrm{CA}$, this procedure may not be suitable for all patients, with $\mathrm{AF}$ recurrence continuing to be observed in one third of the patients undergoing CA [10-12]. Better delineation of AF substrate could lead to improvement in our ability to identify those at risk of recurrent $\mathrm{AF}$. We assessed the prognostic ability of pre-procedural regional atrial myocardial strain and strain rate (SR) imaging using 2D speckle tracking echocardiography (STE) to identify regional atrial functional abnormalities and predictors of arrhythmia recurrence in patients undergoing $\mathrm{CA}$.

\section{Methods}

\subsection{Patient population}

One hundred and forty-five consecutive patients who underwent CA for symptomatic drug refractory AF at the Mayo Clinic Arizona between January 2005 and April 2009 were followed prospectively. All patients who had a baseline transthoracic echocardiogram (TTE) performed during sinus rhythm at our institution before the CA procedure with preserved left ventricular (LV) function (ejection fraction $>55 \%$ ) and a follow-up TTE $>3$ months postablation were included. Age- and gender-matched healthy subjects with preserved LV function and no history of AF undergoing an echocardiogram were used as controls. Data were collected according to Mayo Institutional Review Board requirements of those patients who provided informed consent for chart review. All patients underwent clinical evaluation including 12-lead electrocardiogram (ECG), chest multislice computed tomography, and transesophageal echocardiography. AF was considered paroxysmal if episodes were selfterminating, persistent if required chemical or electric cardioversion for termination, or permanent if electric cardioversion failed or $\mathrm{AF}$ was present continuously for at least 12 months [13].

\subsection{Echocardiographic studies}

Echocardiographic studies were performed prior to ablation and during sinus rhythm using Acuson Sequoia (Siemens Medical, Mountain View, CA) and Vivid-7 (GE Healthcare, Milwaukee, WI) equipment, according to the recommendations of the American Society of Echocardiography [14]. LV ejection fraction (LVEF) by 2D echocardiography was obtained by modified biplane Simpson's method from apical four- and two-chamber views. LV dimensions and wall thickness were made in parasternal long axis with M-mode cursor positioned just beyond the mitral leaflet tips, perpendicular to the long axis of the ventricle. For assessment of LA volume, orthogonal apical views - apical four- and two-chamber views-were obtained to determine LA area and length (from the middle of the plane of the mitral annulus to the posterior wall). The apical long-axis view was then used instead of the two-chamber view if the left atrium in the latter view appeared foreshortened. Specifically, the maximal LA chamber area and length were measured at end-ventricular systole, excluding the LA appendage and pulmonary veins. Left atrial volume was calculated on the basis of the algorithm $([0.85 \times \mathrm{A} 1 \times \mathrm{A} 2] / L)$, where A1 is the four-chamber LA area, A2 is the two-chamber or apical long-axis LA area, and $L$ is the average of the two lengths obtained from the orthogonal views and indexed to body surface area. Indexed LA diameter, fourchamber LA area, and indexed LA volume were categorized according to current American Society of Echocardiography guidelines [14]. The mitral flow velocities were recorded with pulsed-wave Doppler with the sample volume placed at the tip of the mitral valve tips from the apical four-chamber view. From the mitral valve inflow velocity curve, peak E-wave velocity and its deceleration time, and peak A-wave velocity were measured. Tissue 
Doppler-derived peak LV relaxation velocities were obtained from the lateral and septal corners of mitral valve annulus during early $\left(E_{\mathrm{a}}\right)$ and late $\left(A_{\mathrm{a}}\right)$ diastolic phases of the cardiac cycle at a speed of $100 \mathrm{~mm} / \mathrm{s}$.

\subsection{Two-dimensional speckle tracking echocardiography}

Echocardiographic images were stored in digital cineloop format (frame rate $>30$ frames per second; Prosolv Cardiovascular Solutions, Indianapolis, IN) for off-line analysis by customized 2D Cardiac Performance Analysis software (2D CPA, TomTec, Munich, Germany) that employs STE technology for angle-independent measures of 2D strain [15]. This software tracks the local features by finding the motion that maximizes the local frameto-frame correlations subject to the constraint that the motion be periodic, with the period equal to the R-R interval. Iterative 1D correlations were applied in orthogonal directions to improve the speed of the algorithm while still determining 2D motion. To ensure spatial coherence in the endocardial tracking, the software employs a three-point median filter and a three-point Gaussian filter for the displacement computed at neighboring points. Myocardial motion was detected from a user-defined tracing along the LA border in apical four-chamber view throughout one cardiac cycle by successive application of a series of tracking steps. From this motion, LA velocity, longitudinal strain (LS), and SR were calculated along the trace during different phases of the cardiac cycle, and differences in atrial function were determined by real-time quantitative assessment of regional myocardial deformation in sinus rhythm [15].

The LA myocardium was divided into six equidistant regions for obtaining LS curves along the lateral and septal walls adjoining the mitral annulus, mid-atrial walls, and superior or "roof" region corresponding to the area that encompasses the region around the pulmonary veins. All regions were similar in size, and in each region LS was color-coded and displayed as a function of time throughout the cardiac cycle [16]. Strain and SR measurements were performed from the septal and lateral walls of the LA as depicted in Fig. 1. Peak regional and global LS was measured at the end of LV ejection, while LA SR was measured as peak systolic, during early and late diastolic (atrial contraction) LV filling. LA volume curve was generated with the software by continuous tracing of the LA myocardium and calculating LA volumes frame by frame using the single-plane Simpson method. LA maximal $\left(\mathrm{LA}_{\mathrm{vol} \mathrm{max}}\right)$, minimal $\left(\mathrm{LA}_{\mathrm{vol} \mathrm{min}}\right)$, and total emptying fraction $\left(\mathrm{LA}_{\mathrm{vol} \mathrm{max}}-\mathrm{LA}_{\mathrm{vol} \mathrm{min}}\right) /$ $\left.\mathrm{LA}_{\mathrm{vol} \mathrm{max}} \times 100\right)$ were calculated [17].

\subsection{Catheter ablation}

Anti-arrhythmic agents were discontinued at least five half-lives (except amiodarone) and oral anticoagulation stopped 3 days before CA. Low-molecular-weight heparin was administered in high-risk patients until the day before ablation. Catheters were introduced percutaneously through the femoral and right internal jugular veins. Heparin was administered intravenously to maintain an active clotting time $>300 \mathrm{~s}$. A 5-F decapolar catheter (Torqr; inter-electrode spacing 2-5-2 mm; Medtronic Inc., MN) was advanced through the right internal jugular vein into the coronary sinus. A 10-F, 64-element, 5.5- to 10.5-MHz, phased-array intracardiac echocardiography catheter introduced via the left femoral vein was advanced to the right atrium [18] to facilitate transeptal puncture using a Brockenbrough needle along with a transeptal sheath (8-F SL0, St. Jude Medical, Minnetonka, MN) [18]. Ablation was guided by a Lasso catheter positioned at the ostium of the PV under ICE and fluoroscopic guidance. CA was performed by an 8-F 4- to 5-mm Steerable or NaviStar ThermoCool Irrigated Tip Catheter (Biosense Webster, Diamond Bar, CA). LA geometry was rendered by electro-anatomic mapping (CARTO, Biosense-Webster, Diamond Bar, CA, or by Ensite NavX 3D mapping system) and wide area circumferential ablation (WACA) performed to encircle the left- and right-sided PV 5-15 mm outside their 
ostia. Power and temperature setting were set up to $50 \mathrm{~W}$ targeting a temperature of 50$55^{\circ} \mathrm{C}$ with non-irrigated tip catheter and maximum power of $30 \mathrm{~W}$ with irrigated tip catheters. After WACA, electrical isolation of all PVs was confirmed during coronary sinus pacing and entrance and exit block was used as the endpoint of ablation. Additional lines were made to connect the left and right WACA lines and to isolate the lateral mitral isthmus in patients with persistent AF. Cavo-tricuspid isthmus ablation was performed with development of bidirectional conduction block as the endpoint, if typical atrial flutter was induced.

\subsection{Follow-up and clinical outcomes}

Patients were monitored overnight and discharged within $24 \mathrm{~h}$, unless there was a procedural complication. All patients received subcutaneous weight-adjusted low-molecular-weight heparin while being orally anti-coagulated with warfarin to therapeutic INR (between 2 and 3) [18]. Anti-arrhythmic agents were not resumed unless there was early recurrence of AF and, if started, continued for 3 months unless required long term. Follow-up consisted of scheduled visits (at 3, 6, and 12 months and or communication with the patient's primary physician), ECGs, 24-h Holter monitor, and a TTE obtained at 3 months or following recurrence of symptoms. Transient episodes of arrhythmia recurrence within the first 90 days after ablation were not counted as recurrence (blanking period) [10] due to irritability of the atria immediately following CA. Patients presenting with no symptomatic AF episodes or irregular pulse while not on any anti-arrhythmic agent after the blanking period were placed in the "No recurrence group" (NR). Recurrence was defined as the presence of irregularly irregular pulse and AF on rhythm monitoring, AF persistence after the blanking period, or requiring anti-arrhythmic therapy for maintenance of sinus rhythm, and these patients were assigned to the "AF recurrence group" (AFR).

\subsection{Statistical analysis}

Continuous variables were summarized as means with standard deviation. Discrete variables were described as counts with percentages. The differences between AFR, NR, and non-AF group were tested using Kruskal-Wallis test and Wilcoxon rank sum test for continuous variables and chi-square test or Fisher's exact test (if $n \leq 5$ ) for categorical variables. Univariate logistic regression analysis was used to assess the effect of baseline clinical characteristics, echocardiographic, and strain parameters on AF recurrence. Variables with a $p$ value $<0.1$ on univariate analysis were used for multivariate binary logistic regression analysis to calculate the odds ratio with $95 \%$ confidence interval (CI) and to examine the predictive effect of each factor on the risk of AF recurrence. Inter-observer and intraobserver variability of LA STE parameters was assessed in ten patients from each group in duplicate by two observers for longitudinal LA strain. Both observers were blinded to the study data and analysis was performed on the same day in two separate settings. BlandAltman plot and linear regression methods were used to assess the reproducibility. Statistical analyses were performed using SAS version 9.2 (SAS Institute Inc., Cary, North Carolina) and $p<0.05$ was considered to be statistically significant

\section{Results}

\subsection{Patient characteristics}

Baseline clinical characteristics of 63 patients that met the inclusion criteria and were followed for a mean period of $18 \pm 12$ months are summarized in Table 1. Patients were divided into two groups based on clinical AF recurrence (AFR group) and no recurrence (NR group). Fifty-four percent $(n=34)$ of the study population was in sinus rhythm on last follow-up and demonstrated clinical success, while 46\% $(n=29)$ had recurrence. There were no significant differences in age, sex, presence of underlying structural heart disease, 
hypertension, diabetes mellitus, sleep apnea, and ablation strategy between the two groups at baseline. Patients who had AF recurrence compared to NR group had a higher prevalence of hyperlipidemia ( $69 \%$ vs. $44 \%, p=0.04$ ) and the use of beta-blockers ( $89 \%$ vs. $67 \%, p=0.03$ ).

Out of 63 patients undergoing ablation, 44 underwent pulmonary vein isolation (PVI) and 19 WACA, with an equal distribution between the AF recurrence and no recurrence groups (Table 1). No difference was observed in the rate of AF recurrence in the group ablated by WACA vs. PVI ( $47 \%$ vs. $45.5 \% p=0.89)$.

\subsection{Comparison of atrial dimensions, strain, and SR}

Both AFR and NR groups had similar 2D echocardio-graphic and Doppler features, including LA volumes, LV mass index, LVEF, and other parameters of systolic and diastolic function, as summarized in Table 2. Strain parameters compared between AFR, NR, and non-AF groups are summarized in Table 3 . The LA emptying fraction was significantly reduced in the AFR group $(55 \pm 16 \%, p<0.001)$ compared to NR $(64 \pm 14 \%)$ or non-AF group (78 $\pm 9 \%)$. Overall, global and regional strain, SR, and longitudinal velocity during LV ejection (reservoir phase) and early diastole (conduit phase) was markedly diminished in patients who had AF recurrence (Table 3).

\subsection{Predictors of AF recurrence}

The baseline clinical characteristics and 2D and Doppler echo parameters were not predictive of AF recurrence after CA. Various strain and SR parameters with odds ratio, 95\% CI, and $p$ values predictive of AF recurrence are summarized in Table 4. The reduction in lateral wall annular LS and early diastolic SR were predictive of AF recurrence $(p<0.05)$, whereas reduced global and regional systolic SR at five of the six segments showed a trend toward AF recurrence without reaching statistical significance. On multivariate analysis of univariate predictors with $p<0.1$, reduction in lateral wall LS was the only independent predictor of AF recurrence (odds ratio $=1.15,95 \% \mathrm{CI}=1.03-1.3, p=0.01$ ). The pre-ablation LA lateral wall LS in the overall ablation group was significantly reduced (Fig. 2) in those who had AF recurrence (AFR=11.4 \pm 7.0$)$ vs. those that did not have recurrence $(\mathrm{NR}=20.2 \pm 14.2)$ and the non-AF group (23.4 \pm 7.8 , Table 2$)$.

Lateral wall LS values were then compared between patients with paroxysmal and persistent AF. Within the paroxysmal AF population $(n=47)$, LS continued to differentiate those with AF recurrence (11.7 \pm 7$)$ and NR $(19.6 \pm 14.5, p=0.01)$, whereas in patients with persistent AF $(n=16)$, a trend toward reduced strain values was observed in the AFR group (11 \pm 7$)$ when compared to the NR group $(22 \pm 12, p=0.10)$, but did not reach statistical significance.

The receiver operating characteristic curve for lateral wall LS demonstrated the clinical utility of measuring LS for pre-procedural identification of patients at risk of AF recurrence (Fig. 3, area under the curve $=0.70,95 \% \mathrm{CI}=0.57-0.83, p=0.007$ ). Inter- and intra-observer variability data are presented in Table 5 . In the linear regression model, the adjusted $R^{2}$ value ranged from 0.80 to 0.94 . In the Bland-Altman plot, the mean differences [19] were not significant.

\section{Discussion}

The main finding of our study is that in patients referred for CA of symptomatic drug refractory AF, baseline global and regional LA deformation determined by the myocardial strain pattern identified the group who had AF recurrence. Atrial deformation properties measured during ventricular systole and early diastole correlating to an impaired atrial reservoir and conduit function discriminated patients who were unable to maintain sinus rhythm after CA from those who demonstrated clinical success. Atrial dimensions, 
ventricular function, and baseline clinical characteristics were not predictive of maintenance of sinus rhythm in this population with well-preserved LV function. Analysis of the LA strain enabled us to identify patients with apparently advanced AF substrate, particularly in those with paroxysmal AF. Patients with lower strain and SR values seem to have a greater likelihood for $\mathrm{AF}$ recurrence after $\mathrm{CA}$, indicating altered atrial properties compared to those who maintained sinus rhythm. LA lateral wall regional LS was the only independent predictor of AF recurrence and could be helpful in identifying abnormal atrial substrate and predicting ablation outcome.

$\mathrm{CA}$ is increasingly used for the management of patients with symptomatic drug refractory AF [10]. Although highly effective in two thirds of the patients, a substantial minority continues to have recurrent AF [10]. Previously, clinical factors such as older age, presence of atrial enlargement, longer duration, and non-paroxysmal nature of AF have been associated with recurrence of AF in some but not all studies [20-22]. In our study of patients with well-preserved LV function, the mean LA volume and volume index, although increased, was not different between the AFR and NR group and was not predictive of AF recurrence. Atrial deformation depicted as strain and SR reflects atrial compliance and could provide an insight into the relationship between atrial reservoir function and susceptibility to $\mathrm{AF}$, as previously suggested [16, 23, 24]. We examined atrial myocardial deformation reflected by longitudinal shortening and SR measuring the tissue velocity gradient along the LA septal and lateral walls as a prognostic determinant of AF recurrence post-ablation. Reduction in baseline LA lateral wall LS was a more sensitive marker of abnormal atrial substrate than LA enlargement identifying the group that had AF recurrence, particularly those with paroxysmal AF. The numbers of patients with persistent AF were fewer, and despite showing a trend toward reduced LS values in the AFR group, statistical significance was not reached. During ventricular systole, LA serves as a reservoir to store blood when $\mathrm{AV}$ valves are closed. This reservoir function is influenced by atrial relaxation and chamber stiffness $[16,23,24]$, which suggests that despite similar atrial dimensions, patients with $\mathrm{AF}$ recurrence had a more advanced substrate abnormality as indicated by depressed systolic strain and SR.

Regional atrial functional abnormalities have been demonstrated to be associated with heterogeneities in electrical conduction [25] that predicts recurrence of AF after cardioversion [1, 4]. Strain and SR imaging using tracking of acoustic kernels by 2D STE [15], a novel angle-independent tool for measuring myocardial stretch with the ability to quantitatively analyze global and regional LA contractility and relaxation, could therefore serve as a noninvasive tool to identify contractile dysfunction overcoming the existing limitations of Doppler-based technologies, including the effect of tethering on intrinsic myocardial function [26-30].

Recently, strain and SR have been recognized as useful predictors of AF recurrence after CA. [31-37]. Hwang et al. [37], in a younger population (mean age 54 \pm 9 years) with paroxysmal AF, and Reant et al. [31], in isolated paroxysmal AF, demonstrated strain and SR imaging to predict success of CA. We extend these observations to a patient population who were older and included both paroxysmal and persistent AF with common comorbidities such as hypertension, sleep apnea, obesity, and diabetes, typically present in AF patients encountered in clinical practice [1]. Schneider et al. [32], using atrial strain and SR immediately following CA, demonstrated that higher values correlated with maintenance of sinus rhythm over the 3-month follow-up. Although strain values after CA were useful to predict AF recurrence and indicate the utility of this tool, what is more practically desirable is definition of the atrial substrate pre-procedurally to predict long-term success beyond 3 months, typically considered a period during which atrial arrhythmias could arise due to increased irritability and inflammation of the atria from the ablation lesions [10], 
independent of the underlying substrate abnormalities. LA scarring could also be estimated by invasive approaches, such as intra-cardiac voltage mapping [33] or noninvasive 3D delayed gadolinium enhancement MRI [34], both shown to predict recurrence of AF after ablation demonstrating a relationship with atrial substrate abnormalities. Despite its utility in identifying atrial fibrosis, voltage mapping has limited clinical usefulness pre-procedurally for the majority of patients due to invasive nature requiring placement of intra-cardiac catheters in a specialized laboratory [5]. Similarly, MRI is not readily available, requires a specialized facility, is expensive, and lacks the resolution that can accurately identify scattered fibrosis within the thin-walled atria [35]. A readily available, inexpensive, and noninvasive method of assessing atrial substrate abnormality that provides prognostic information about the success of CA procedure is highly desirable [5]. In this regard, the value of 2D STE arises from its ability to directly measure wall deformation [15] that can be used at the bedside and provide additional information about atrial function to what can be obtained from a conventional cavity-based echocardiographic analysis [14, 36].

Limitations to our study include a relatively small sample size due to stringent inclusion criteria. Additionally, patients were not randomized to PVI or WACA and the choice of procedure was left to the discretion of the operating physician; therefore, differences in outcome could be due to variations in technique. However, the number of patients that underwent WACA or PVI between the groups was similar and no difference in AF recurrence was observed. A further limitation was that strain and SR parameters might be influenced by pre-load and post-load conditions attributable to differences in medical therapy and heterogeneity in the patient population. However, this concern was minimized by the lack of differences between the groups in the number of patients treated with diuretics, angiotensin-converting enzyme inhibitors, or angiotensin receptor antagonists. The possibility that a decrease in strain could be due to increased wall stress cannot be entirely excluded. The extent of global and regional substrate abnormalities that are critical for the increased predisposition to AF recurrence and associated complications are not fully characterized, and further prospective studies with long-term longitudinal follow-up are warranted to help stratify patient subgroups that can be selected with the highest anticipated likelihood of success from an intervention.

In summary, we report subtle abnormalities in atrial function that can be detected by $2 \mathrm{D}$ STE as predictive of recurrence of AF after apparently successful CA. Characterization of abnormal atrial myocardial substrate by this noninvasive modality may aid to appropriately select patients being considered for AF ablation who will benefit most from this expensive and potentially risky procedure [5]. Additional prospective studies will be required to reinforce and further these findings.

\section{Acknowledgments}

This study was supported in part by National Heart, Lung, and Blood Institute R01 HL101240-01 grant. Dr. Jahangir is also supported by R01 HL089542-03.

\section{Abbreviations}

$\begin{array}{ll}\text { AF } & \text { Atrial fibrillation } \\ \text { AFR } & \text { Atrial fibrillation recurrence } \\ \text { CA } & \text { Catheter ablation } \\ \text { NR } & \text { No recurrence group } \\ \text { SR } & \text { Strain rate }\end{array}$




\section{References}

1. Fuster V, Ryden LE, Cannom DS, Crijns HJ, Curtis AB, Ellenbogen KA, et al. ACC/AHA/ESC 2006 Guidelines for the Management of Patients with Atrial Fibrillation: a report of the American College of Cardiology/American Heart Association Task Force on Practice Guidelines and the European Society of Cardiology Committee for Practice Guidelines (Writing Committee to Revise the 2001 Guidelines for the Management of Patients With Atrial Fibrillation): developed in collaboration with the European Heart Rhythm Association and the Heart Rhythm Society. Circulation. 2006; 114:e257-e354. [PubMed: 16908781]

2. Lloyd-Jones D, Adams RJ, Brown TM, Carnethon M, Dai S, De Simone G, et al. Heart disease and stroke statistics-2010 update. A report from the American Heart Association. Circulation. 2010; 121(7):e46-e215. [PubMed: 20019324]

3. Park TH, Nagueh SF, Khoury DS, Kopelen HA, Akrivakis S, Nasser K, et al. Impact of myocardial structure and function postinfarction on diastolic strain measurements: Implications for assessment of myocardial viability. American Journal of Physiology. Heart and Circulatory Physiology. 2006; 290:H724-H731. [PubMed: 16183729]

4. Nattel S, Shiroshita-Takeshita A, Brundel BJ, Rivard L. Mechanisms of atrial fibrillation: Lessons from animal models. Progress in Cardiovascular Diseases. 2005; 48:9-28. [PubMed: 16194689]

5. Cappato R, Calkins H, Chen SA, Davies W, Iesaka Y, Kalman J, et al. Updated worldwide survey on the methods, efficacy, and safety of catheter ablation for human atrial fibrillation. Circulation: Arrhythmia and Electrophysiology. 2010; 3:32-38. [PubMed: 19995881]

6. Khan MN, Jais P, Cummings J, Di Biase L, Sanders P, Martin DO, et al. Pulmonary-vein isolation for atrial fibrillation in patients with heart failure. New England Journal of Medicine. 2008; 359:1778-1785. [PubMed: 18946063]

7. Pappone C, Rosanio S, Oreto G, Tocchi M, Gugliotta F, Vicedomini G, et al. Circumferential radiofrequency ablation of pulmonary vein ostia: A new anatomic approach for curing atrial fibrillation. Circulation. 2000; 102:2619-2628. [PubMed: 11085966]

8. Wazni OM, Marrouche NF, Martin DO, Verma A, Bhargava M, Saliba W, et al. Radiofrequency ablation vs antiarrhythmic drugs as first-line treatment of symptomatic atrial fibrillation: A randomized trial. JAMA. 2005; 293:2634-2640. [PubMed: 15928285]

9. Wilber DJ, Pappone C, Neuzil P, De Paola A, Marchlinski F, Natale A, et al. Comparison of antiarrhythmic drug therapy and radiofrequency catheter ablation in patients with paroxysmal atrial fibrillation: A randomized controlled trial. JAMA. 2010; 303:333-340. [PubMed: 20103757]

10. Calkins H, Reynolds MR, Spector P, Sondhi M, Xu Y, Martin A, et al. Treatment of atrial fibrillation with antiarrhythmic drugs or radiofrequency ablation: Two systematic literature reviews and meta-analyses. Circulation: Arrhythmia and Electrophysiology. 2009; 2:349-361. [PubMed: 19808490]

11. Tzou WS, Marchlinski FE, Zado ES, Lin D, Dixit S, Callans DJ, et al. Long-term outcome after successful catheter ablation of atrial fibrillation. Circulation: Arrhythmia and Electrophysiology. 2010; 3:237-242. [PubMed: 20335557]

12. Arya A, Piorkowski C, Sommer P, Kottkamp H, Hindricks G. Clinical implications of various follow up strategies after catheter ablation of atrial fibrillation. Pacing and Clinical Electrophysiology. 2007; 30:458-462. [PubMed: 17437567]

13. Jahangir A, Lee V, Friedman PA, Trusty JM, Hodge DO, Kopecky SL, et al. Long-term progression and outcomes with aging in patients with lone atrial fibrillation: A 30-year follow-up study. Circulation. 2007; 115:3050-3056. [PubMed: 17548732]

14. Lang RM, Bierig M, Devereux RB, Flachskampf FA, Foster E, Pellikka PA, et al. Recommendations for chamber quantification: a report from the American Society of Echocardiography's Guidelines and Standards Committee and the Chamber Quantification Writing Group, developed in conjunction with the European Association of Echocardiography, a branch of the European Society of Cardiology. Journal of the American Society of Echocardiography. 2005; 18:1440-1463. [PubMed: 16376782]

15. Geyer H, Caracciolo G, Abe H, Wilansky S, Carerj S, Gentile F, et al. Assessment of myocardial mechanics using speckle tracking echocardiography: Fundamentals and clinical applications. 
Journal of the American Society of Echocardiography. 2010; 23:351-369. (quiz 453-455). [PubMed: 20362924]

16. Inaba Y, Yuda S, Kobayashi N, Hashimoto A, Uno K, Nakata T, et al. Strain rate imaging for noninvasive functional quantification of the left atrium: Comparative studies in controls and patients with atrial fibrillation. Journal of the American Society of Echocardiography. 2005; 18:729-736. [PubMed: 16003270]

17. Jarnert C, Melcher A, Caidahl K, Persson H, Ryden L, Eriksson MJ. Left atrial velocity vector imaging for the detection and quantification of left ventricular diastolic function in type 2 diabetes. European Journal of Heart Failure. 2008; 10:1080-1087. [PubMed: 18838296]

18. Cha YM, Friedman PA, Asirvatham SJ, Shen WK, Munger TM, Rea RF, et al. Catheter ablation for atrial fibrillation in patients with obesity. Circulation. 2008; 117:2583-2590. [PubMed: 18474813]

19. Bland JM, Altman DG. Statistical methods for assessing agreement between two methods of clinical measurement. Lancet. 1986; 1:307-310. [PubMed: 2868172]

20. Berruezo A, Tamborero D, Mont L, Benito B, Tolosana JM, Sitges M, et al. Pre-procedural predictors of atrial fibrillation recurrence after circumferential pulmonary vein ablation. European Heart Journal. 2007; 28:836-841. [PubMed: 17395676]

21. Oral H, Knight BP, Tada H, Ozaydin M, Chugh A, Hassan S, et al. Pulmonary vein isolation for paroxysmal and persistent atrial fibrillation. Circulation. 2002; 105:1077-1081. [PubMed: 11877358]

22. Balk EM, Garlitski AC, Alsheikh-Ali AA, Terasawa T, Chung M, Ip S. Predictors of atrial fibrillation recurrence after radiofrequency catheter ablation: A systematic review. Journal of Cardiovascular Electrophysiology. 2010; 21:1208-1216. [PubMed: 20487117]

23. Hoit BD. Assessing atrial mechanical remodeling and its consequences. Circulation. 2005; 112:304-306. [PubMed: 16027269]

24. Hoit BD, Walsh RA. Regional atrial distensibility. The American Journal of Physiology. 1992; 262:H1356-H1360. [PubMed: 1590438]

25. Kistler PM, Sanders P, Fynn SP, Stevenson IH, Spence SJ, Vohra JK, et al. Electrophysiologic and electroanatomic changes in the human atrium associated with age. Journal of the American College of Cardiology. 2004; 44:109-116. [PubMed: 15234418]

26. Yip G, Abraham T, Belohlavek M, Khandheria BK. Clinical applications of strain rate imaging. Journal of the American Society of Echocardiography. 2003; 16:1334-1342. [PubMed: 14652617]

27. Fang ZY, Yuda S, Anderson V, Short L, Case C, Marwick TH. Echocardiographic detection of early diabetic myocardial disease. Journal of the American College of Cardiology. 2003; 41:611617. [PubMed: 12598073]

28. Urheim S, Edvardsen T, Torp H, Angelsen B, Smiseth OA. Myocardial strain by Doppler echocardiography. Validation of a new method to quantify regional myocardial function. Circulation. 2000; 102:1158-1164. [PubMed: 10973846]

29. Voigt JU, Flachskampf FA. Strain and strain rate. New and clinically relevant echo parameters of regional myocardial function. Zeitschrift für Kardiologie. 2004; 93:249-258.

30. D'Andrea A, De Corato G, Scarafile R, Romano S, Reigler L, Mita C, et al. Left atrial myocardial function in either physiological or pathological left ventricular hypertrophy: A two-dimensional speckle strain study. British Journal of Sports Medicine. 2008; 42:696-702. [PubMed: 18070810]

31. Reant P, Lafitte S, Bougteb H, Sacher F, Mignot A, Douard H, et al. Effect of catheter ablation for isolated paroxysmal atrial fibrillation on longitudinal and circumferential left ventricular systolic function. The American Journal of Cardiology. 2009; 103:232-237. [PubMed: 19121442]

32. Schneider C, Malisius R, Krause K, Lampe F, Bahlmann E, Boczor S, et al. Strain rate imaging for functional quantification of the left atrium: Atrial deformation predicts the maintenance of sinus rhythm after catheter ablation of atrial fibrillation. European Heart Journal. 2008; 29:1397-1409. [PubMed: 18436560]

33. Verma A, Wazni OM, Marrouche NF, Martin DO, Kilicaslan F, Minor S, et al. Pre-existent left atrial scarring in patients undergoing pulmonary vein antrum isolation: An independent predictor of procedural failure. Journal of the American College of Cardiology. 2005; 45:285-292. [PubMed: 15653029] 
34. Kuppahally SS, Akoum N, Burgon NS, Badger TJ, Kholmovski EG, Vijayakumar S, et al. Left atrial strain and strain rate in patients with paroxysmal and persistent atrial fibrillation: Relationship to left atrial structural remodeling detected by delayed enhancement-MRI. Circulation. Cardiovascular Imaging. 2010; 3:231-239. [PubMed: 20133512]

35. Mastouri R, Sawada SG, Mahenthiran J. Current noninvasive imaging techniques for detection of coronary artery disease. Expert Review of Cardiovascular Therapy. 2010; 8:77-91. [PubMed: 20030023]

36. Pieper GM, Shah A, Harmann L, Cooley BC, Ionova IA, Migrino RQ. Speckle-tracking 2dimensional strain echocardiography: A new noninvasive imaging tool to evaluate acute rejection in cardiac transplantation. Journal of Heart and Lung Transplantation. 2010; 29:1039-1046. [PubMed: 20488730]

37. Hwang HJ, Choi EY, Rhee SJ, Joung B, Lee BH, Lee SH, et al. Left atrial strain as predictor of successful outcomes in catheter ablation for atrial fibrillation: A two-dimensional myocardial imaging study. Journal of Interventional Cardiac Electrophysiology. 2009; 26:127-132. [PubMed: 19529886] 
(a)

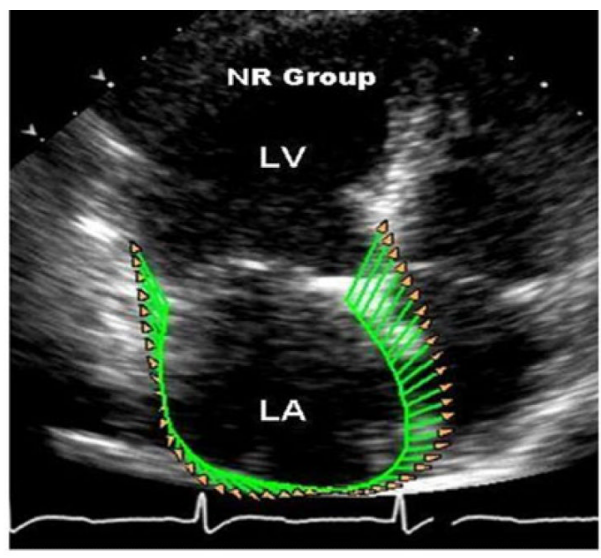

(c)

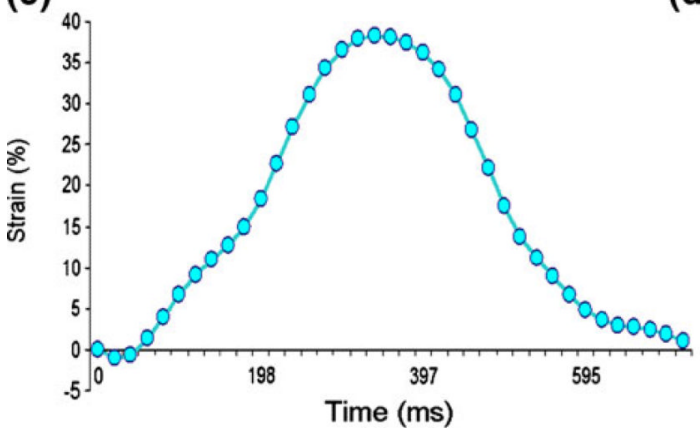

(e)

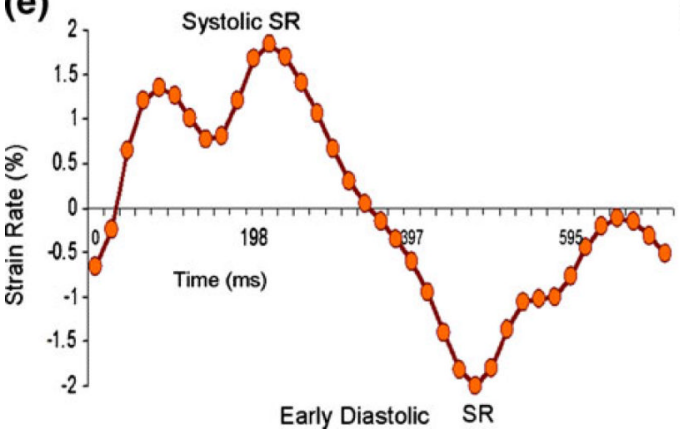

(b)

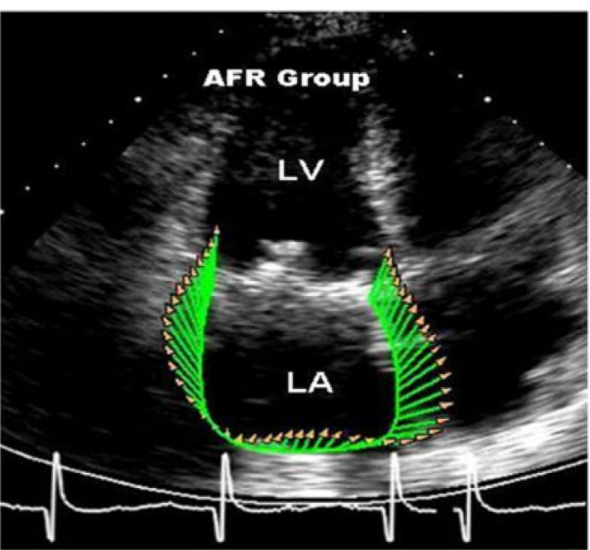

(d)

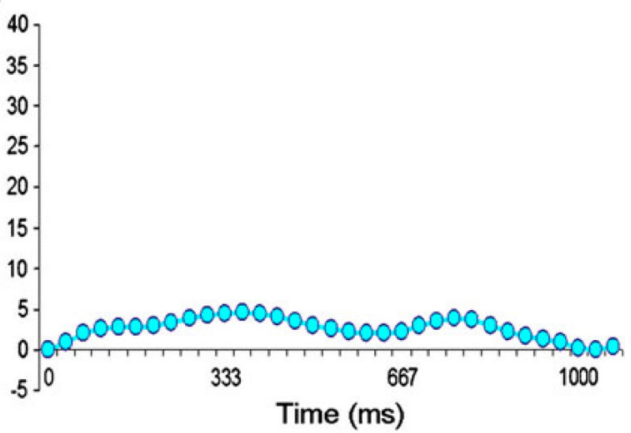

(f)

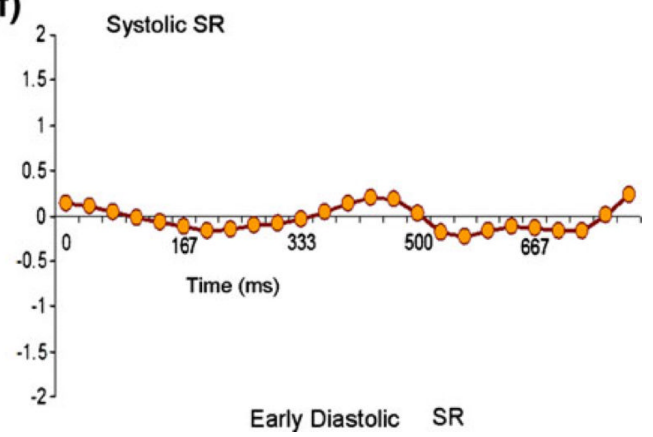

Fig. 1.

Assessment of LA strain by speckle tracking echocardiography. Left atrial wall was tracked in apical four-chamber view using speckle tracking echocardiography. $(\mathbf{a}, \mathbf{b})$ Tracking of LA wall and corresponding velocity vectors in a patient without recurrence and with recurrence of AF. (c, d) Corresponding global strains. (e, f) Global SR in the two patients. Note the diminished global strain and SR in the patient who had recurrence of AF. $L A$ left atrial; $A F$ atrial fibrillation; $A F R$ atrial fibrillation recurrence; $N R$ no recurrence group 


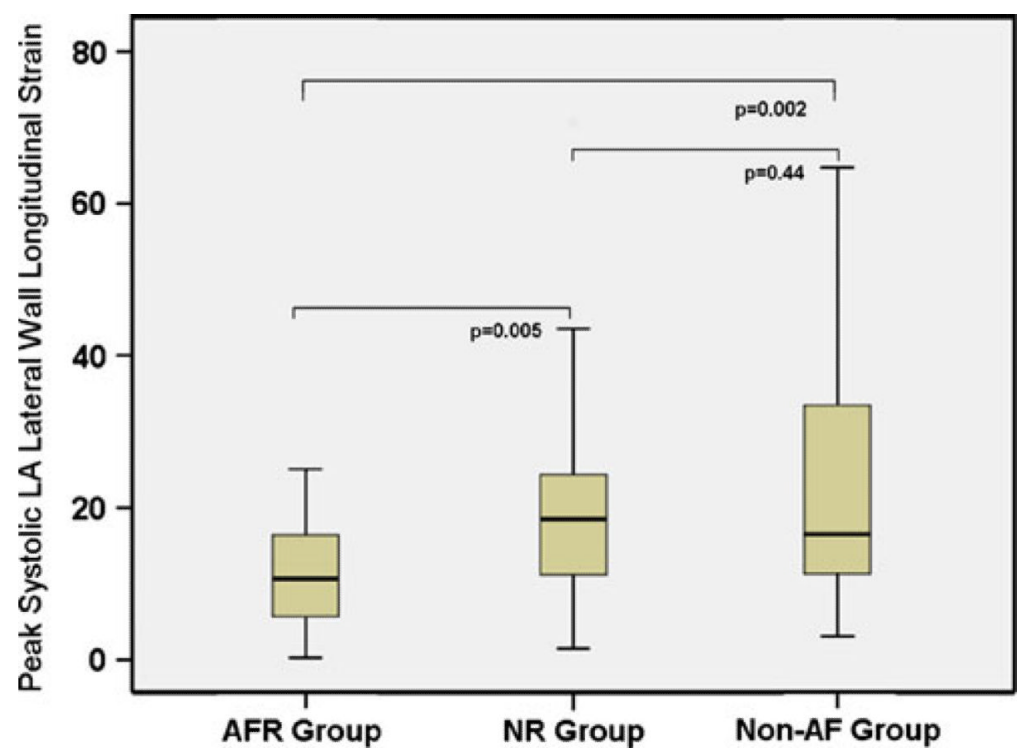

Fig. 2.

Box plot showing the differences in LA lateral wall LS as an independent predictor of recurrence 


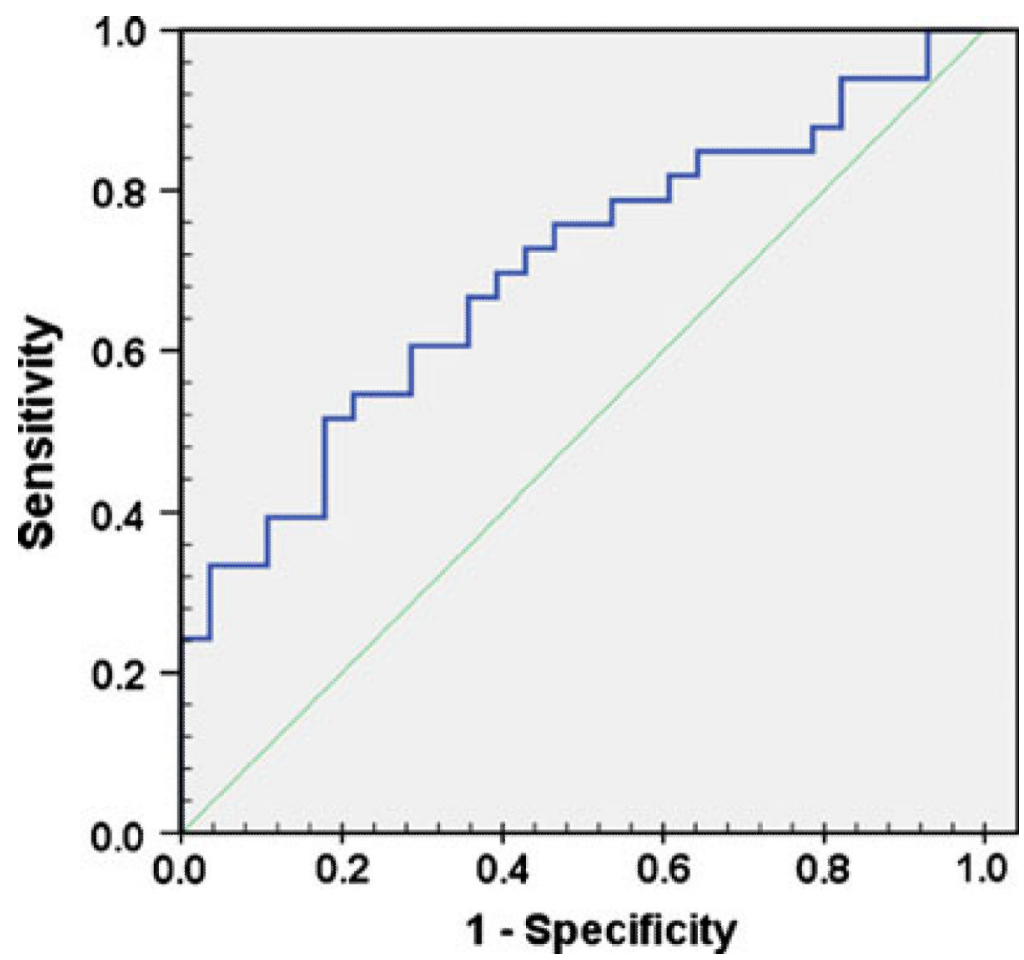

Fig. 3.

ROC curve for the prognostic utility of LA lateral wall LS 
Table 1

Clinical characteristics of atrial fibrillation recurrence (AFR) and no recurrence (NR) groups at baseline

\begin{tabular}{|c|c|c|c|c|}
\hline Characteristic & Overall & AFR group & NR group & $p$ value \\
\hline$N$ & 63 & $29(46.0 \%)$ & $34(54.0 \%)$ & \\
\hline Age (years) & $63.1 \pm 9.9$ & $65.0 \pm 8.7$ & $61.6 \pm 10.7$ & 0.178 \\
\hline Female & $25(39.7 \%)$ & $11(37.9 \%)$ & $14(41.2 \%)$ & 0.793 \\
\hline AF: Paroxysmal & $47(74.6 \%)$ & $18(62 \%)$ & $29(85.3 \%)$ & 0.688 \\
\hline Persistent & $16(25 \%)$ & $11(38 \%)$ & $5(14.7 \%)$ & 0.23 \\
\hline Structural heart disease & $2(3.2 \%)$ & $2(6.9 \%)$ & $0(0.0 \%)$ & 0.208 \\
\hline Coronary artery disease & $7(11.1 \%)$ & $3(10.3 \%)$ & $4(11.8 \%)$ & 1 \\
\hline Congestive heart failure & $1(1.6 \%)$ & $0(0.0 \%)$ & $1(2.9 \%)$ & 1 \\
\hline CVA/TIA & $3(4.7 \%)$ & $2(6.9 \%)$ & $1(2.9 \%)$ & 0.8 \\
\hline Hypertension & $33(53.2 \%)$ & $16(57.1 \%)$ & $17(50.0 \%)$ & 0.876 \\
\hline Diabetes mellitus & $9(14.3 \%)$ & $4(13.8 \%)$ & $5(14.7 \%)$ & 1 \\
\hline Hyperlipidemia & $35(55.6 \%)$ & $20(69.0 \%)$ & $15(44.1 \%)$ & 0.048 \\
\hline Obesity & $8(12.7 \%)$ & $5(17.2 \%)$ & $3(8.8 \%)$ & 0.317 \\
\hline Obstructive sleep apnea & $18(28.6 \%)$ & $11(37.9 \%)$ & $7(20.6 \%)$ & 0.129 \\
\hline Coumadin & $43(68.3 \%)$ & $23(79.3 \%)$ & $20(58.8 \%)$ & 0.082 \\
\hline Aspirin & $27(42.9 \%)$ & $13(44.8 \%)$ & $14(41.2 \%)$ & 0.77 \\
\hline Statins & $25(39.7 \%)$ & $14(48.3 \%)$ & $11(32.4 \%)$ & 0.198 \\
\hline Ca channel blockers & $21(33.9 \%)$ & $9(31.0 \%)$ & $12(36.4 \%)$ & 0.658 \\
\hline Beta-blockers & $49(77.8 \%)$ & $26(89.7 \%)$ & $23(67.7 \%)$ & 0.036 \\
\hline Anti-arrhythmic agents & $61(97 \%)$ & $29(100 \%)$ & $32(94 \%)$ & 0.2 \\
\hline \multicolumn{5}{|l|}{ Ablation procedure } \\
\hline PVI & $44(70 \%)$ & $20(69 \%)$ & $24(70.5 \%)$ & 0.68 \\
\hline WACA & $19(30 \%)$ & $9(31 \%)$ & $10(29.5 \%)$ & 0.68 \\
\hline
\end{tabular}

J Interv Card Electrophysiol. Author manuscript; available in PMC 2012 September 1. 
Table 2

Echo characteristics at baseline

\begin{tabular}{lclll}
\hline Characteristic & Overall & Atrial fibrillation recurrence (AFR) group & No recurrence (NR) group & $\boldsymbol{p}$ value \\
\hline$N$ & 63 & $29(46.0 \%)$ & $34(54.0 \%)$ & \\
LA volume index & $33.7 \pm 10.1$ & $34.8 \pm 11.4$ & $32.9 \pm 9$ & 0.688 \\
LV mass index & $86.8 \pm 19.1$ & $87.4 \pm 21.4$ & $86.4 \pm 17.5$ & 0.959 \\
LV diastolic vol & $90.0 \pm 31.3$ & $92.4 \pm 34.4$ & $87.6 \pm 28.4$ & 0.766 \\
LV systolic vol & $34.8 \pm 15.4$ & $38.1 \pm 17.7$ & $31.5 \pm 12.3$ & 0.323 \\
LVEF (\%) & $63.5 \pm 5.7$ & $62.4 \pm 5.6$ & $64.4 \pm 5.7$ & 0.229 \\
E/e' medial & $11.2 \pm 7.2$ & $12.2 \pm 10$ & $10.5 \pm 4.2$ & 0.903 \\
E/e' lateral & $8.3 \pm 3.9$ & $8.6 \pm 4.3$ & $8.0 \pm 3.5$ & 0.904 \\
\hline
\end{tabular}


$m$
$\frac{0}{0}$
$\frac{10}{10}$

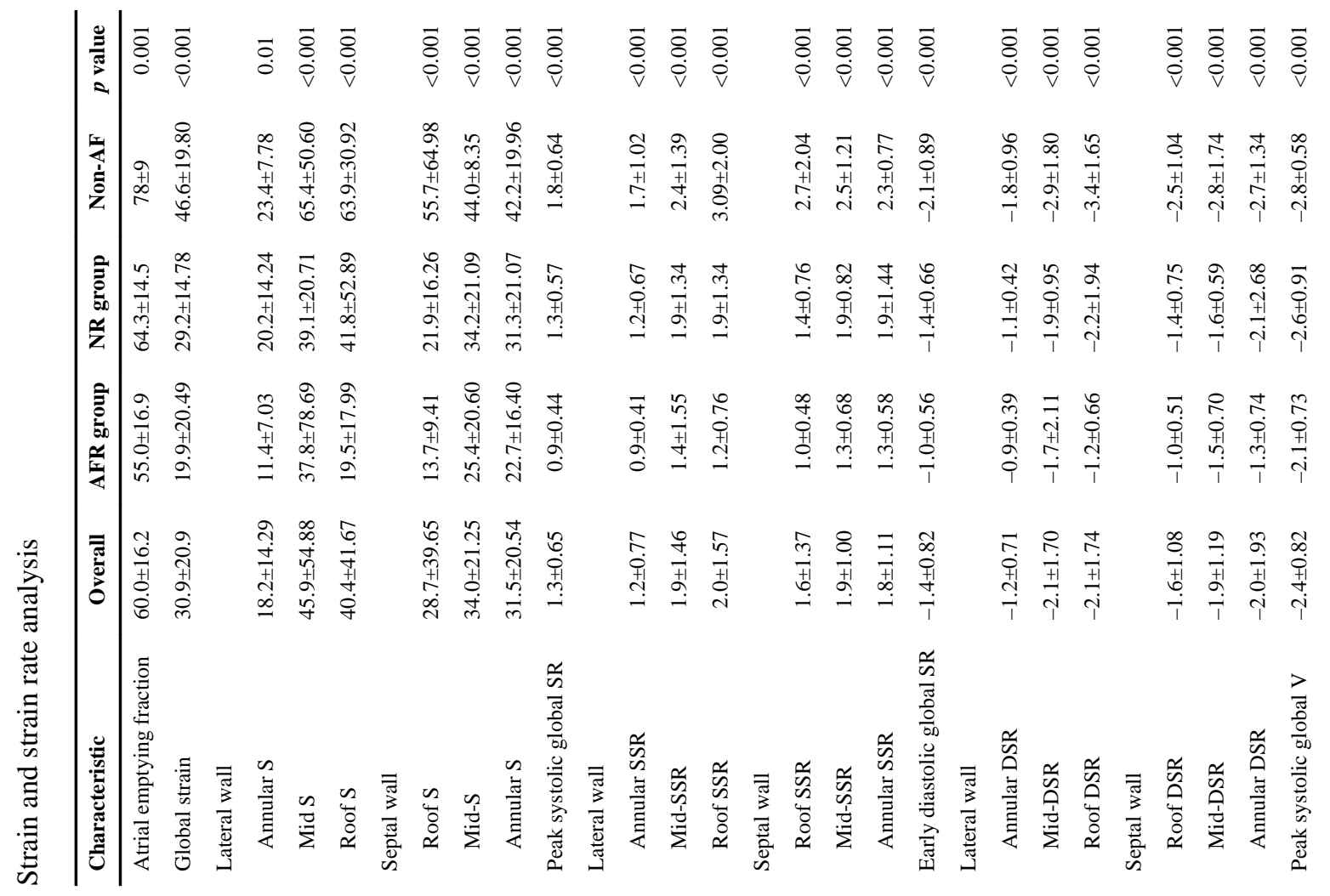




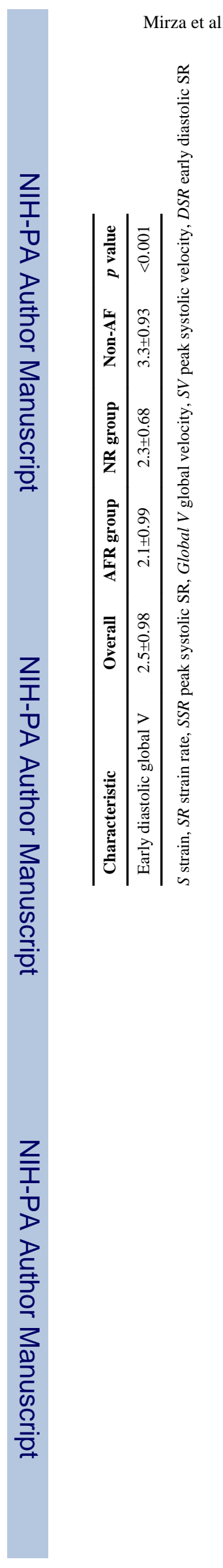

Page 17

J Interv Card Electrophysiol. Author manuscript; available in PMC 2012 September 1. 
Table 4

Univariate strain predictors of $\mathrm{AF}$ recurrence after $\mathrm{CA}$

\begin{tabular}{|c|c|c|}
\hline Characteristic & Odds ratio $(95 \%$ CI $)$ & $p$ value \\
\hline Global strain & $0.985(0.968-1.003)$ & 0.097 \\
\hline \multicolumn{3}{|l|}{ Lateral wall } \\
\hline Annular S & $0.970(0.942-0.998)$ & 0.036 \\
\hline Mid-S & $0.998(0.991-1.006)$ & 0.664 \\
\hline Roof S & $0.986(0.971-1.001)$ & 0.065 \\
\hline \multicolumn{3}{|l|}{ Septal wall } \\
\hline Roof S & $0.976(0.953-1.000)$ & 0.053 \\
\hline Mid-S & $0.990(0.976-1.004)$ & 0.152 \\
\hline Annular S & $0.988(0.973-1.004)$ & 0.133 \\
\hline Peak systolic global SR & $0.676(0.440-1.037)$ & 0.073 \\
\hline \multicolumn{3}{|l|}{ Lateral wall } \\
\hline Annular SSR & $0.683(0.440-1.061)$ & 0.090 \\
\hline Mid-SSR & $0.850(0.666-1.085)$ & 0.193 \\
\hline Roof SSR & $0.770(0.580-1.024)$ & 0.072 \\
\hline \multicolumn{3}{|l|}{ Septal wall } \\
\hline Roof SSR & $0.722(0.494-1.056)$ & 0.093 \\
\hline Mid-SSR & $0.781(0.585-1.041)$ & 0.092 \\
\hline Annular SSR & $0.774(0.582-1.031)$ & 0.080 \\
\hline Early diastolic global SR & $1.370(0.929-2.019)$ & 0.112 \\
\hline \multicolumn{3}{|l|}{ Lateral wall } \\
\hline Annular DSR & $1.398(0.873-2.238)$ & 0.163 \\
\hline Mid-DSR & $1.077(0.868-1.337)$ & 0.500 \\
\hline Roof DSR & $1.308(1.001-1.708)$ & 0.049 \\
\hline \multicolumn{3}{|l|}{ Septal wall } \\
\hline Roof DSR & $1.354(0.930-1.969)$ & 0.114 \\
\hline Mid-DSR & $1.164(0.863-1.570)$ & 0.319 \\
\hline Annular DSR & $1.245(0.939-1.652)$ & 0.128 \\
\hline
\end{tabular}

J Interv Card Electrophysiol. Author manuscript; available in PMC 2012 September 1. 


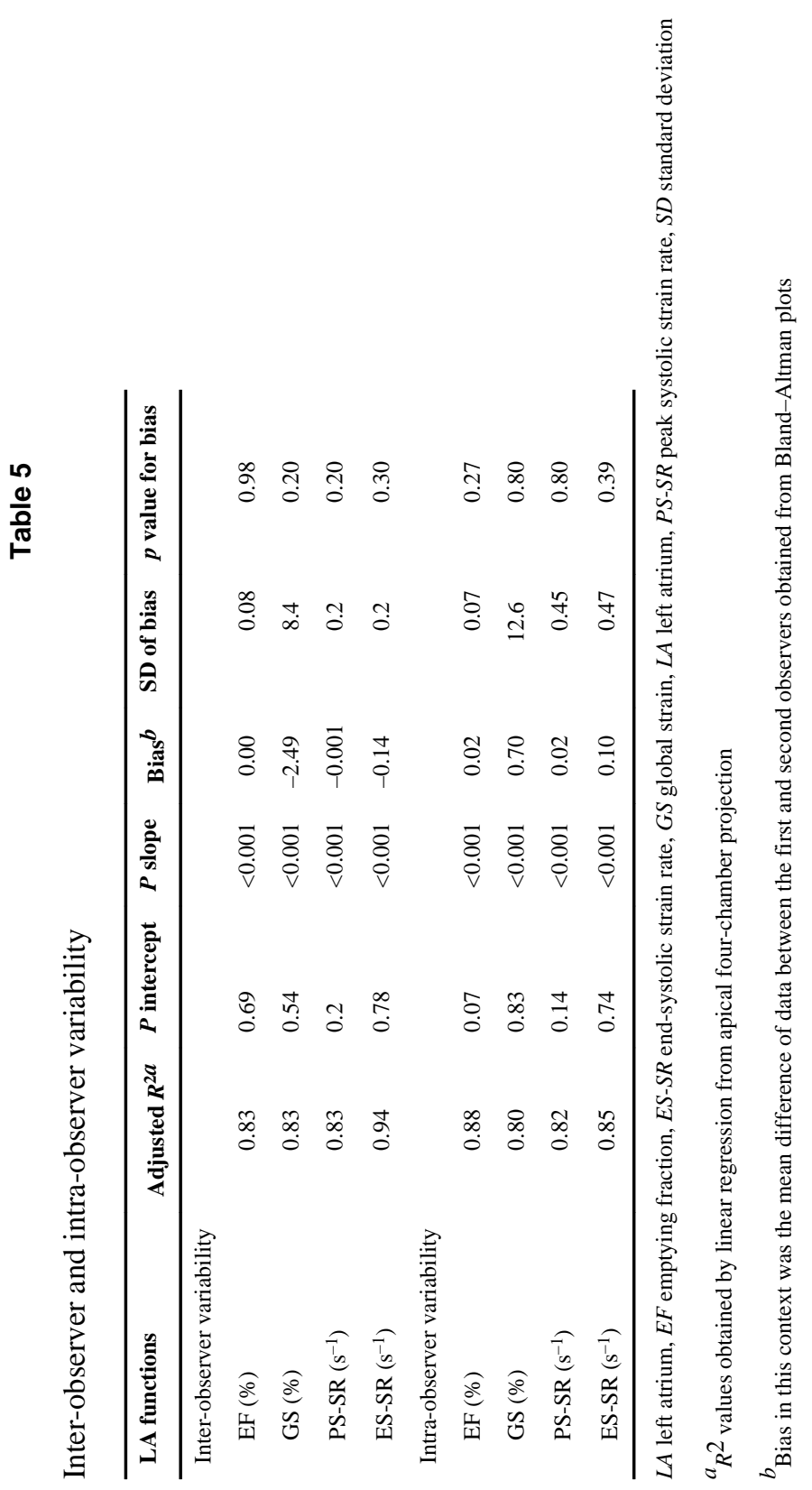

J Interv Card Electrophysiol. Author manuscript; available in PMC 2012 September 1. 